\title{
Wireless Local Area Network Signal Strength Measurement for Sensor Localization without New Anchors
}

\author{
Shigemi Ishida, ${ }^{1 *}$ Yutaka Arakawa, ${ }^{1}$ Shigeaki Tagashira, ${ }^{2}$ and Akira Fukuda ${ }^{1}$ \\ ${ }^{1}$ Graduate School/Faculty of Information Science and Electrical Engineering, Kyushu University, \\ 744 Moto-oka, Nishi-ku, Fukuoka-shi, Fukuoka 819-0395, Japan \\ ${ }^{2}$ Faculty of Informatics, Kansai University, \\ 2-1-1 Ryozenji-cho, Takatsuki-shi, Osaka 569-1095, Japan
}

(Received September 5, 2019; accepted October 29, 2019)

Keywords: wireless local area network (WLAN) anchors, sensor localization, cross-technology signal detection

In sensor networks, sensor localization is one of the mandatory functions for physical sensing. In this paper, we propose a sensor localization system that utilizes wireless local area network (WLAN) access points (APs) as anchor nodes. WLAN is widely available nowadays, and WLAN APs are used in many environments. Therefore, for our localization system, we utilize the WLAN APs already installed in the environment, thereby eliminating the installation of new anchors. We present an AP received signal strength (RSS) measurement method, which is the key technology of our sensor localization system. Notably, sensor nodes equipped with an IEEE 802.15.4 (ZigBee) module cannot detect WLAN AP signals. We therefore developed an AP signal detection method with AP identification by employing a cross-technology signal detection technique. Experimental evaluations demonstrate that our AP signal detection method successfully identified sender APs with a false positive (FP) detection rate less than $20 \%$. The experiments also validated that the measured AP-RSS followed the same distribution as the RSS measured using a WLAN device.

\section{Introduction}

The sensor network, which is used in not only outdoor but also indoor environments, is one of the fundamental components in many Internet of Things (IoT) systems. In many IoT systems, sensor localization is a mandatory function because a sensor network is used for physical sensing.

The location of sensor nodes is either generally estimated using the global positioning system (GPS) or manually measured. Therefore, while building a large-scale indoor sensor network in which the GPS is unavailable, we need to measure the location of a large number of sensors. Human activity monitoring, home energy management, and building energy management systems are typical examples of large-scale indoor sensor networks.

To address the sensor localization problem, indoor localization systems have been studied. ${ }^{(3-5)}$ These studies mainly focused on deployment cost reduction ${ }^{(6-15)}$ or accuracy improvement ${ }^{(16-21)}$ *Corresponding author: e-mail: ishida@ait.kyushu-u.ac.jp Conference papers ${ }^{(1,2)}$ containing preliminary results of this paper appeared in VTC 2015-Fall and CANDAR 2015. https://doi.org/10.18494/SAM.2020.2592 
related to localization systems. The methods presented in these studies have successfully reduced the deployment cost or improved accuracy with the help of either users or manually measured anchor nodes.

The aim of the present study is to develop an indoor sensor localization system that enables us to eliminate the deployment of new anchors. To this end, we propose a sensor localization system that uses wireless local area network (WLAN) access points (APs) as anchor nodes. WLAN is now prevalent, and WLAN APs are already used in many indoor environments. We measure the received signal strength (RSS) of multiple WLAN APs by using a sensor node and estimate the sensor's location using the RSS.

The sensor nodes, however, are equipped with an IEEE 802.15.4 (ZigBee; although ZigBee and IEEE 802.15.4 are different wireless technologies, we use the word ZigBee instead of IEEE 802.15.4 in this paper for readability) module that cannot detect WLAN AP signals. Therefore, to realize the proposed sensor localization system, detecting the WLAN AP signal and measuring its RSS by using a sensor node is the key technology.

We therefore present a method of measuring the RSS of WLAN AP signals by using a sensor node. To overcome differences in wireless technologies, we employ a simple folding signal processing technique presented in $\mathrm{ZiFi}^{(22)}$ and extract $\mathrm{AP}$ beacon signals. An AP identification method is also developed because sensor localization requires both the sender AP information and the RSS. We conducted the experiments in an office environment and observed that our WLAN AP-RSS measurement method successfully measured the RSS of multiple WLAN AP signals while identifying the sender APs as well.

The contributions of this paper are as follows.

- We propose an indoor sensor localization system that eliminates additional anchor deployment by using WLAN APs, which are already widely available in indoor environments.

- We develop a WLAN AP-RSS measurement method using a sensor node equipped with a ZigBee radio module, which is the key technology to realize our sensor localization system.

- We propose an AP signal detection method based on the concept of the beacon interval. We show both theoretically and experimentally that the AP signal detection method accurately identifies the sender APs.

- We experimentally demonstrate that the AP-RSS measurement method successfully measured the AP-RSS to confirm the feasibility of the proposed sensor localization system.

The remainder of this paper is organized as follows. Section 2 gives an overview of the new sensor localization system and its design challenges. Section 3 presents the details of the AP-RSS measurement method, followed by the theoretical analysis of the AP signal detection method in Sect. 4. In Sect. 5, we report experimental evaluations. Section 6 presents related work and Sect. 7 concludes this paper.

\section{Sensor Localization System Utilizing WLAN APs as Anchors}

Figure 1 depicts the overview of the sensor localization system that utilizes WLAN APs. The localization system utilizes the WLAN APs already installed in the environment as anchor nodes. The sensor nodes measure the RSS of the AP signals and send their RSS information 


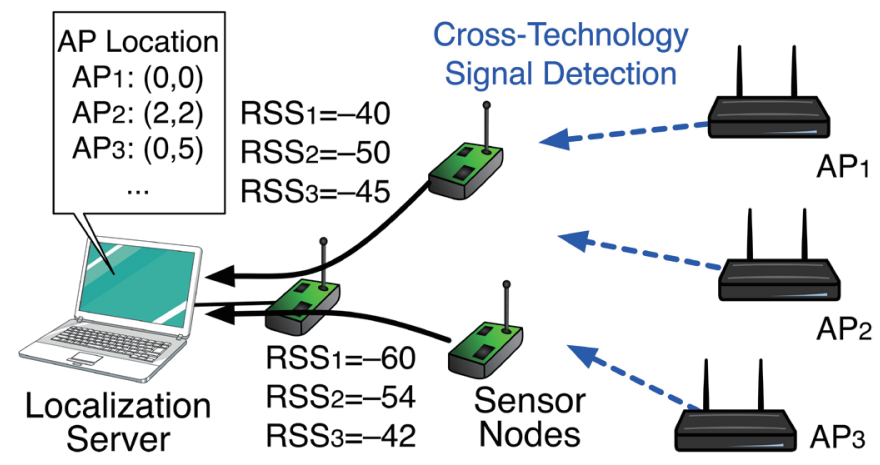

Fig. 1. (Color online) Sensor localization system utilizing WLAN APs as anchors.

to a localization server, which then estimates the location of the APs by using an existing localization algorithm such as trilateration. For AP localization, the required AP information such as the location of APs is collected on the localization server prior to using the sensor localization system.

To realize the sensor localization system, we face the following two major challenges:

(1) AP-RSS measurement on sensor node:

Sensor nodes are equipped with a ZigBee module that cannot detect WLAN signals. Moreover, there are many WLAN devices in indoor environments nowadays. In addition to WLAN devices, the unlicensed $2.4 \mathrm{GHz}$ Industry, Scientific, and Medical (ISM) band is used for implementing many other wireless technologies such as Bluetooth. Therefore, we also need to filter out wireless signals other than WLAN AP signals by using the ZigBee module on a sensor node.

(2) Sender AP identification:

To estimate sensor location, we need AP-RSS information together with the sender AP's information such as location or unique ID. Although we do not limit the localization method used in our localization system, range-based localization requires AP location information. The fingerprinting range-free localization method also needs to identify the sender APs.

In the following section, we present a WLAN AP-RSS measurement method that overcomes the above two challenges.

\section{WLAN AP-RSS Measurement Using Sensor Nodes}

The WLAN AP-RSS measurement method consists of three steps, namely, AP signal detection, AP identification, and AP-RSS extraction. A sensor node periodically samples the RSS of any wireless signal on a specific channel, which is then processed using a simple signal processing technique named folding, which was presented in $\mathrm{ZiFi}{ }^{(22)}$ The folding technique relies on the periodicity of beacon signals sent from WLAN APs. All the APs are configured to send beacon signals at different intervals to perform AP identification. The sensor node finally calculates the average of the RSS samples corresponding to the detected AP signals to derive the AP-RSS. In the following subsections, we present the design details of each step. 


\subsection{AP signal detection}

Figure 2 depicts the AP signal detection process. To detect WLAN AP signals, a sensor node periodically measures the RSS of any wireless signal on a specific ZigBee channel. The IEEE 802.15.4 standard defines an energy detection function, which enables us to measure the RSS of any wireless signal on a specific ZigBee channel. Although WLAN and ZigBee modules use different wireless technologies, they share the same $2.4 \mathrm{GHz}$ band. A ZigBee module therefore detects the WLAN signal energy as well.

Figure 3 depicts a typical example of WLAN signals captured on a ZigBee-compatible MICAz sensor node. Furthermore, ZigBee's energy detection function provides the RSS of the WLAN AP signals averaged over $128 \mu \mathrm{s}$. We therefore sample the RSS every $128 \mu$ s to avoid missing WLAN AP signals while also minimizing the sampling rate to reduce memory usage.

Subsequently, the sensor node determines whether the channel is busy, i.e., occupied, and describes the channel-usage status by 0 (clear) or 1 (busy). We name these $1 / 0$ samples as channel-usage samples. The channel is considered busy when the RSS is greater than or equal to $-77 \mathrm{dBm}$, which is the default threshold of clear channel assessment of a CC2420 IEEE 802.15.4 module. ${ }^{(23)}$

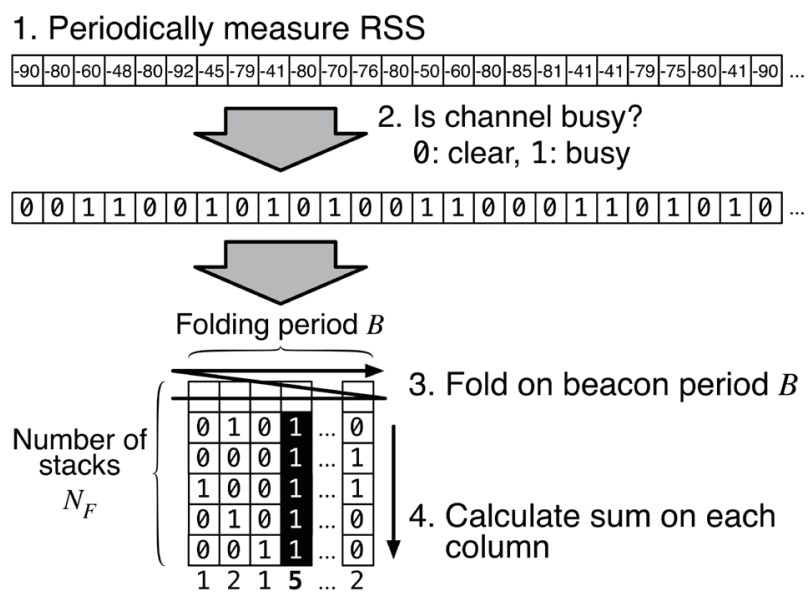

Fig. 2. AP signal detection using folding.

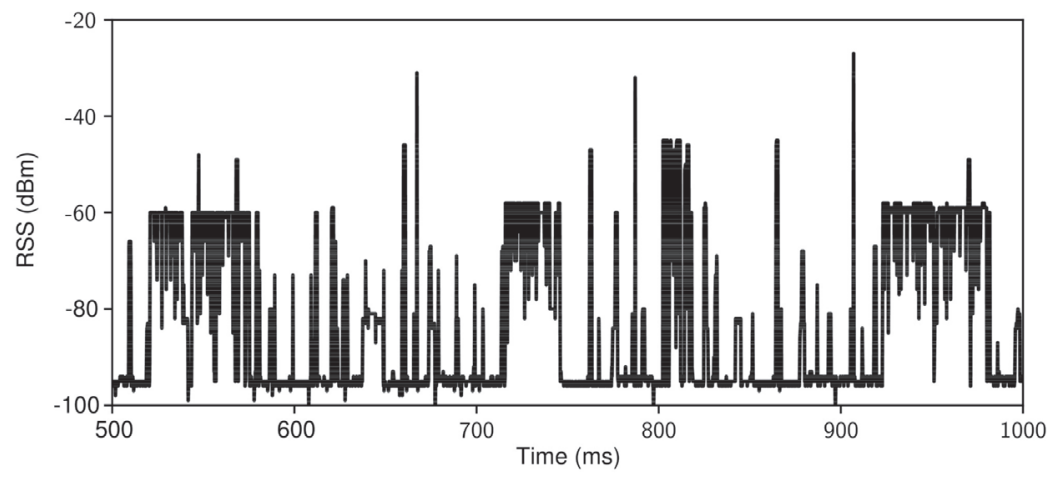

Fig. 3. Example of WLAN signals on ZigBee channel 19 captured using MICAz sensor node. 
We then fold the channel-usage samples at the WLAN AP beacon interval, thereby deriving a channel-usage matrix. The folding period $B$ is calculated from the WLAN AP beacon interval $t$ as

$$
B=t /\left(128 \times 10^{-6}\right),
$$

because every channel-usage sample corresponds to $128 \mu$ s. Finally, the sum of each column of the channel-usage matrix is calculated. The sum is called the channel-usage sum.

WLAN AP beacon signals are detected by finding the columns whose channel-usage sum is greater than the threshold. Periodic WLAN AP beacon signals appear in a column when the beacon interval and folding period are identical. A large channel-usage sum indicates the presence of WLAN AP beacon signals whose interval is identical to the folding period.

For beacon signal detection, we use the overlapping rate instead of the original channelusage sum, which depends on the number of stacks, $N_{F}$, in the channel-usage matrix. The overlapping rate is defined as the ratio of the channel-usage sum to $N_{F}$. When the maximum overlapping rate for a channel-usage matrix is greater than the threshold, beacon signals are detected. The threshold of the overlapping rate is determined by theoretical analysis in Sect. 4 .

\subsection{AP identification}

Using the folding technique presented in Sect. 3.1, a sensor node can detect WLAN AP signals. However, to identify the sender AP on a sensor node, we utilize the beacon multiplexing technique presented in FreeBee. ${ }^{(24,25)}$ WLAN APs are configured using coprime beacon intervals to separately detect WLAN AP beacons that have coprime intervals. AP identification is based on the following theorem.

Theorem 1. Let $t_{A}$ and $t_{B}$ be coprime numbers. Upon designing a channel-usage matrix having folding period $t_{A}$, the beacon signals corresponding to folding period $t_{B}$ appear in the same column every $t_{B}$ rows in the channel-usage matrix.

Proof: In a channel-usage matrix with folding period $t_{A}$, the beacon signals corresponding to folding period $t_{B}$ appear in the same column every $\operatorname{lcm}\left(t_{A}, t_{B}\right)$ samples, where $\operatorname{lcm}\left(t_{A}, t_{B}\right)$ is the least common multiple of $t_{A}$ and $t_{B}$. However, when $t_{A}$ and $t_{B}$ are coprime, $\operatorname{lcm}\left(t_{A}, t_{B}\right)=t_{A} t_{B}$. Since the channel-usage matrix has $t_{A}$ columns, the beacon signals corresponding to folding period $t_{B}$ appear in the same column every $\operatorname{lcm}\left(t_{A}, t_{B}\right) / t_{A}=t_{B}$ rows.

Because $t_{B} \geq 2$, the beacon signals corresponding to folding period $t_{B}$ appear in the same column no more than every two rows in a channel-usage matrix that has folding period $t_{A}$. Theoretically, $t_{B}$ beacon signals are easily filtered out using an overlapping rate greater than 0.5 .

Furthermore, we need to identify multiple WLAN APs for localization. Therefore, WLAN APs are configured using beacon intervals such that any pair of intervals are coprime. The multiples and divisors of 100 time units (TUs, $1 \mathrm{TU}=1024 \mu \mathrm{s}$ ), which are the default beacon intervals in many WLAN APs available on the market, should also be avoided. For multiple APs, the easiest way to select the beacon intervals is to use prime numbers. 
In a practical environment, however, this simple identification technique suffers from false positive (FP) detection because of the asynchronous operations of WLAN APs and sensor nodes. Moreover, a beacon signal larger in time length than one RSS sample might also increase the rate of FP detection because the beacon signals appear in multiple columns in a channel-usage matrix.

To reduce the rate of FP detection in a practical environment, we apply the beacon interval selection algorithm presented in Algorithm 1. The function $P_{f p}(A, B)$ in Algorithm 1 denotes the probability of the beacon signals having interval $A$ being mistakenly detected as beacon signals having interval $B$. The theoretical derivation of the function $P_{f p}(A, B)$ is difficult because of the existence of beacon frames having different lengths and the asynchronous operations of WLAN APs and sensor nodes. However, we experimentally derive $P_{f p}(A, B)$ in Sect. 5.2.

\subsection{AP-RSS extraction}

The AP-RSS extraction block calculates the RSS of the detected AP beacon signals. The detected AP beacon signals correspond to the channel-usage samples in a channel-usage matrix. Intuitively, the AP-RSS is derived by calculating the average of the RSS samples corresponding to the channel-usage samples; however, the calculation is highly erroneous because of a partial averaging problem.

The partial averaging problem is caused by the definition of ZigBee's energy detection function, i.e., RSS measurement function. The ZigBee modules provide the RSS over an averaging window of $128 \mu \mathrm{s}$. However, the transmission of a WLAN AP beacon signal might start and end in the middle of the averaging windows, as depicted in Fig. 4, as we already know that WLAN APs and sensor nodes are not synchronized. The RSS derived within an averaging window including the start and end of beacon signals might suffer from a large error.

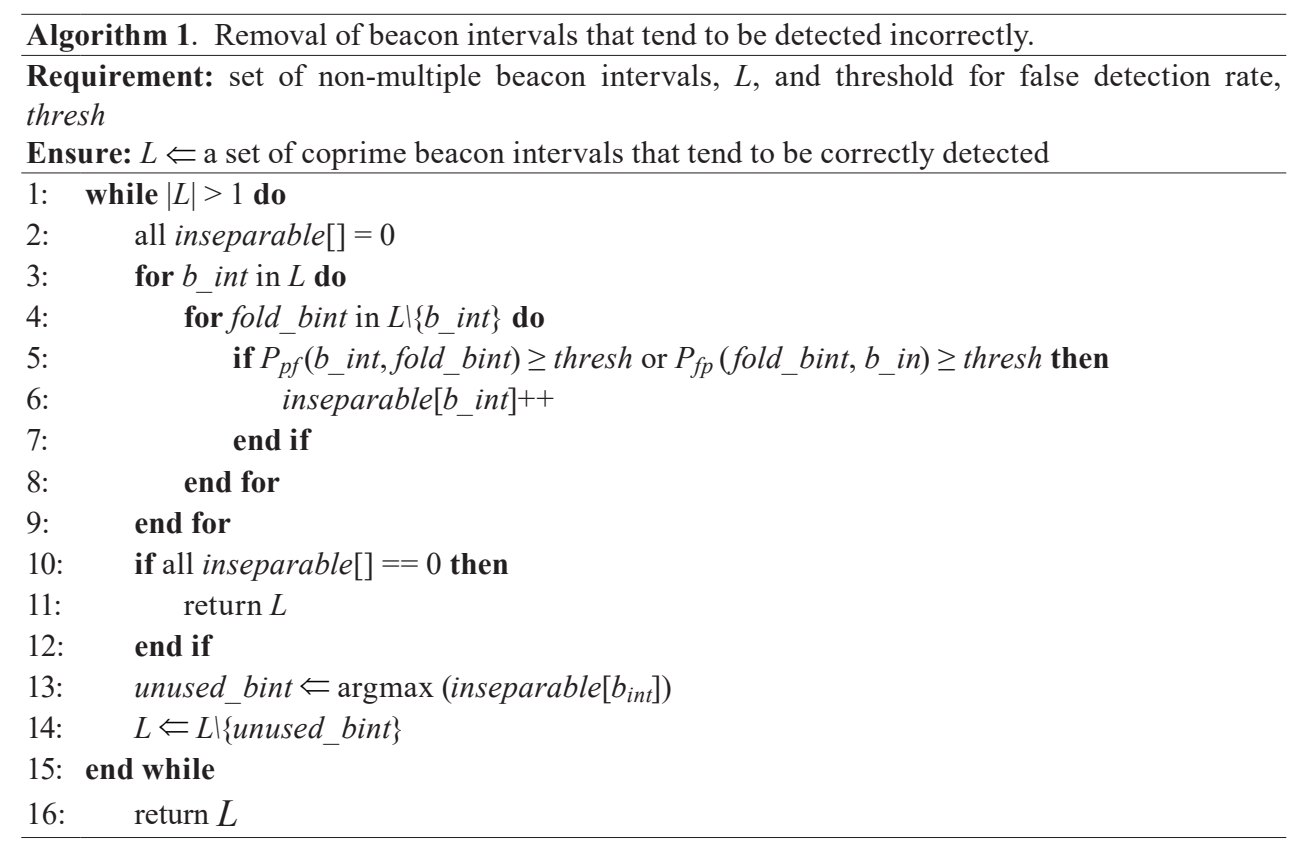




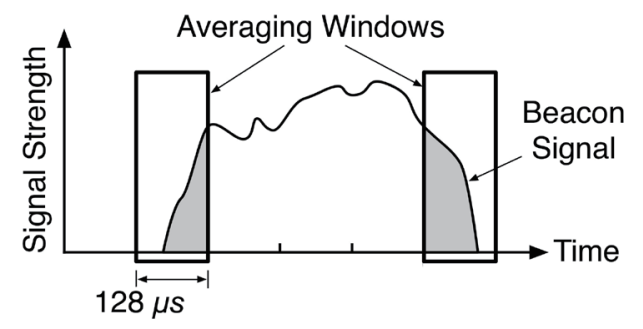

Fig. 4. Partial averaging problem.

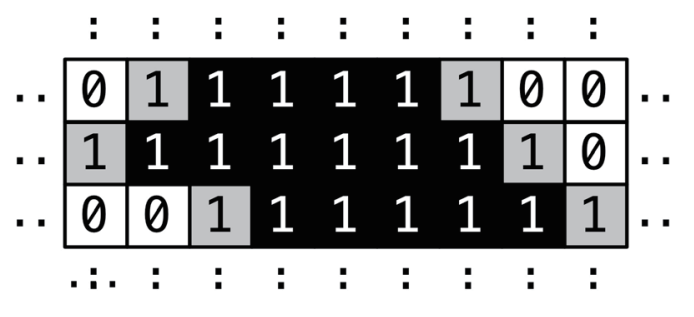

Fig. 5. Example of filtering of channel-usage samples.

To reduce the effect of the partial averaging problem, we apply a simple filter before averaging the RSS samples. We ignore the channel-usage samples including the start and end of the WLAN AP beacon signals. Figure 5 illustrates the columns in a channel-usage matrix in which a beacon signal is detected. In each row, the successive " 1 "s indicate a beacon signal. For AP-RSS calculation, in the channel-usage matrix, we ignore the first and last "1"s, i.e., the gray cells in Fig. 5, because they are likely to be affected by the partial averaging problem. The AP-RSS is therefore calculated by calculating the average of the RSS samples corresponding to the black channel-usage samples in Fig. 5.

To apply this simple filtering technique, the time length of a beacon signal must be longer than that of three RSS samples, i.e., more than $128 \times 3=384 \mu \mathrm{s}$. Figure 6 depicts the format of an IEEE 802.11 beacon frame, i.e., beacon signal. A beacon frame is one of the IEEE 802.11 management frames, consisting of a physical (PHY) header and a medium access control (MAC) frame.

The PHY header comprises a physical layer convergence procedure (PLCP) preamble and a PCLP header. In the $2.4 \mathrm{GHz}$ ISM band, the time length of the PHY header is $192 \mu \mathrm{s}$.

The MAC frame comprises a 24-octet header, a frame body, and a 4-octet frame check sequence. The length of the frame body depends on the contents of the beacon signal. Table 1 presents the mandatory fields in the frame body along with their length. ${ }^{(26)}$ On the basis of Table 1, the minimum length of the frame body is 23 octets. The MAC frame is therefore no smaller than 51 octets.

In the $2.4 \mathrm{GHz}$ ISM band, many WLAN APs operate in an IEEE $802.11 \mathrm{~b}$-compatible mode. The beacon frames are sent at a rate of 1 or $2 \mathrm{Mbps}$. The time length of the beacon frames is therefore larger than $396 \mu \mathrm{s}$ ( $=192 \mu \mathrm{s}+51$ octets $\times 8$ bits $/ 2 \mathrm{Mbps})$, which is greater than $384 \mu \mathrm{s}$.

Figure 7 depicts the empirical cumulative probability of the time length of the beacon frames collected while walking in Kyushu University buildings. We collected the beacon frames from 207 APs. Note that the $X$-axis in Fig. 7 is inverted. The blue and red dashed lines represent the cumulative probability of 0.95 and the beacon-frame time length of $384 \mu \mathrm{s}$, respectively. Figure 7 indicates that $95 \%$ of the APs sent beacon frames having time lengths greater than $384 \mu \mathrm{s}$. Moreover, 10 APs sent beacon frames having time lengths less than $396 \mu$ s. Notably, these APs were operating in an IEEE 802.11g mode. However, our AP-RSS measurement method only supports the APs operating in an IEEE 802.11b-compatible mode, which is a limitation of our method. 


\begin{tabular}{|c|c|c|c|c|c|}
\hline \multirow[t]{2}{*}{$\begin{array}{l}\text { Length } \\
\text { (octets) }\end{array}$} & \multicolumn{3}{|r|}{24} & Variable & 4 \\
\hline & PLCP Preamble & PLCP Header & MAC Header & Frame Body & FCS \\
\hline
\end{tabular}

Fig. 6. IEEE 802.11 beacon frame format.

Table 1

Mandatory fields in frame body of beacon frames. ${ }^{(26)}$

\begin{tabular}{lc}
\hline Item & Length (octets) \\
\hline Timestamp & 8 \\
Beacon Interval & 2 \\
Capability & 2 \\
Service Set Identifier (SSID) & $2-34$ \\
Supported Rates & $3-10$ \\
Traffic Indication Map (TIM) & $6-256$ \\
\hline
\end{tabular}

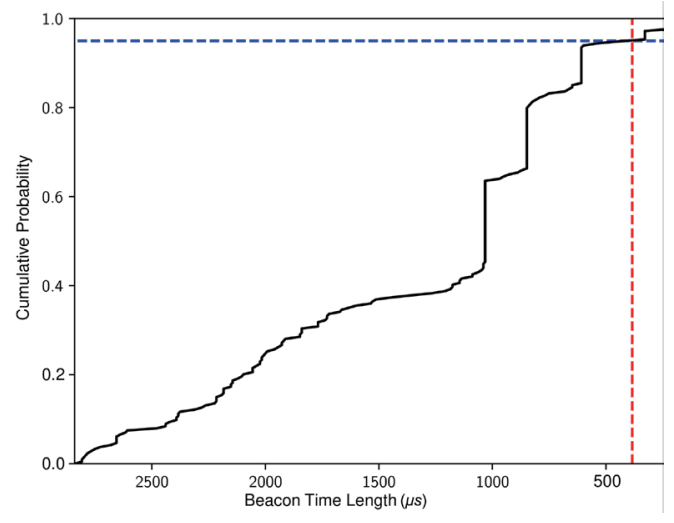

Fig. 7. (Color online) Empirical cumulative probability of beacon-frame time length. Blue and red dashed lines represent the probability of 0.95 and the beacon-frame time length of $384 \mu \mathrm{s}$, respectively.

\section{Theoretical Analysis}

\subsection{Maximum overlapping rate with many APs}

When there are many APs near a sensor node, the number of FP detections increases even upon using coprime beacon intervals. Consider the case in which we try to detect beacon signals whose interval is 2 TU. For multiple APs, we can easily use prime numbers as beacon intervals, as described in Sect. 3.2. In this case, the beacon intervals of the non-target APs are prime numbers greater than 2. When there are two APs having beacon intervals of 3 and $5 \mathrm{TU}$, the beacon signals appear in the same column every 3 and 5 columns in a channel-usage matrix according to Theorem 1. Moreover, note that the beacon signals from both APs overlap in the channel-usage matrix every $15(=3 \times 5)$ rows. The maximum overlapping rate $s_{2}$ caused by the two APs is calculated as

$$
s_{2}=\frac{1}{3}+\frac{1}{5}-\frac{1}{15}=\frac{7}{15}
$$

Similarly, the maximum overlapping rate $s_{n}$ for $n$ APs can be calculated as 


$$
\left\{\begin{array}{c}
s_{1}=\frac{1}{p_{2}} \\
s_{n+1}=s_{n}+\frac{1}{p_{n+1}}-\frac{s_{n}}{p_{n+1}},
\end{array}\right.
$$

where $p_{1}, \ldots, p_{n+1}$ denote the beacon intervals of the non-target APs among the $n$ APs.

Figure 8 depicts the maximum overlapping rate caused by the non-target APs upon using prime numbers as beacon intervals. It depicts two cases, one using the smallest beacon intervals and one using beacon intervals greater than $50 \mathrm{TU}$. As the number of non-target APs increases, the maximum overlapping rate also increases. Upon using the smallest beacon intervals, i.e., 2, 3 , $5,7, \ldots$, the maximum overlapping rate becomes $82.3 \%$ when there are 100 non-target APs.

To reduce the maximum overlapping rate caused by the non-target APs, we use longer beacon intervals. Upon using beacon intervals greater than $50 \mathrm{TU}$, the maximum overlapping rate becomes $37.6 \%$ when there are 100 non-target APs. An overlapping-rate threshold greater than 0.5 easily reduces the number of FP detections in this case.

\subsection{FP detections due to data communication}

In addition to the beacon signals from non-target APs, the AP signal detection on a sensor node also suffers from WLAN data communication. Assume that data communication randomly occurs. Let $N_{F}$ denote the number of rows in a channel-usage matrix. We can calculate the probability that the channel-usage sum of a specific column in a channel-usage matrix becomes $i$ as

$$
\left(\begin{array}{c}
N_{F} \\
i
\end{array}\right) U^{i}(1-U)^{N_{F}-i},
$$

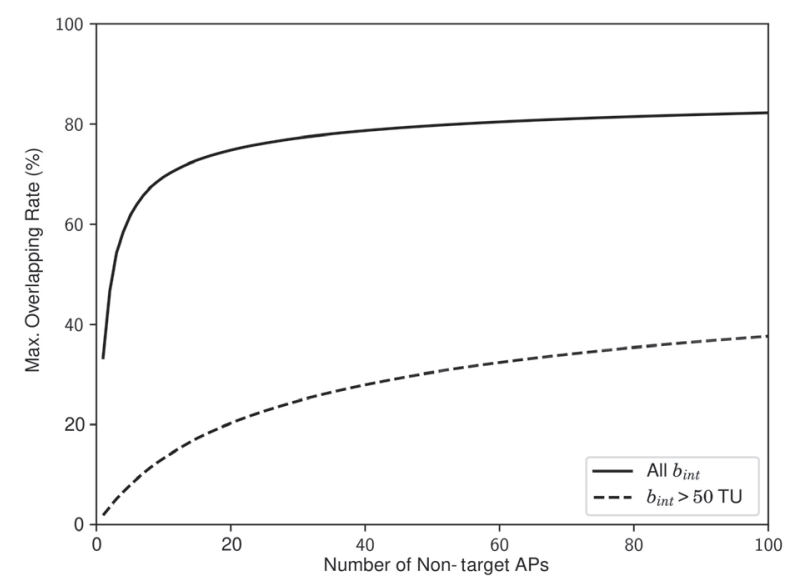

Fig. 8 Maximum overlapping rate as a function of the number of APs. 
where $\left(\begin{array}{l}a \\ b\end{array}\right)$ represents a binomial coefficient and $U$ the rate of channel utilization, i.e., channel occupancy rate, for the WLAN data communication.

Let $X$ be a random variable representing the maximum overlapping rate in a channel-usage matrix and $V_{t h}$ the overlapping-rate threshold. The probability that all the columns in a channelusage matrix have overlapping rates less than the overlapping-rate threshold is calculated using Eq. (4) as

$$
P\left[X<V_{t h}\right]=\left\{\sum_{i=0}^{N_{F} V_{t h}-1}\left(\begin{array}{c}
N_{F} \\
i
\end{array}\right) U^{i}(1-U)^{N_{F}-i}\right\}^{B},
$$

where $B$ denotes the number of columns in the channel-usage matrix. The probability of FP detection caused by data communication is therefore calculated as

$$
P\left[X \geq V_{t h}\right]=1-P\left[X<V_{t h}\right]=1-\left\{\sum_{i=0}^{N_{F} V_{t h}-1}\left(\begin{array}{c}
N_{F} \\
i
\end{array}\right) U^{i}(1-U)^{N_{F}-i}\right\}^{B} .
$$

Figure 9 depicts the rate of FP detection caused by the WLAN data communication as a function of the overlapping rate. In the figure, the rate is calculated using Eq. (6) and $N_{F}=30$. According to Eq. (6), increasing the folding period $B$ increases the number of FP detections. Therefore, $B=500 \mathrm{TU}$ is used in Fig. 9, which is the maximum beacon interval in a practical range. Moreover, the rate of channel utilization is changed from 0.1 to 0.3 because it is less than 0.3 in practical environments. ${ }^{(2)}$ From Fig. 9, it can be observed that using the overlappingrate threshold of $80 \%$ reduces the rate of FP detection to less than $0.01 \%$. Therefore, we use the overlapping-rate threshold of $80 \%$ in our evaluation.

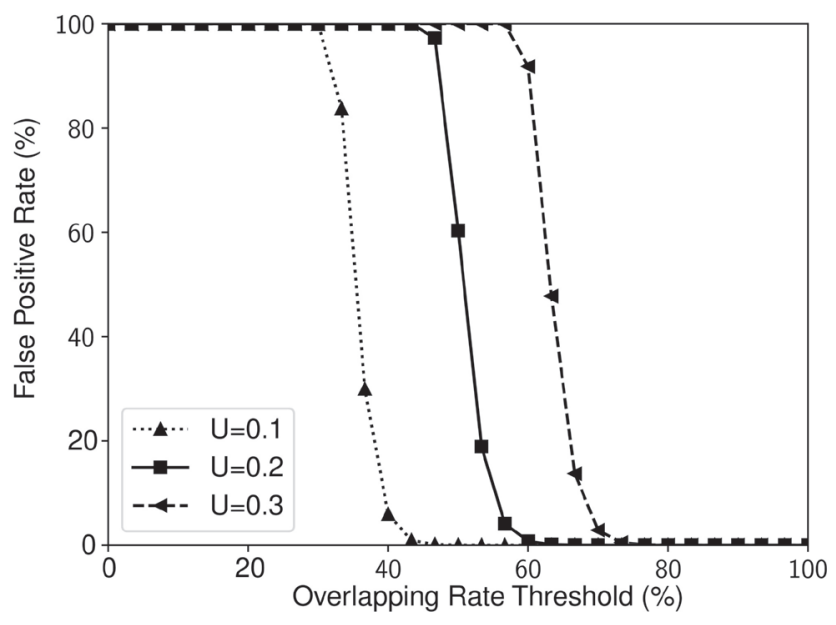

Fig. 9. Rate of FP detection caused by data communication as a function of overlapping-rate threshold (number of foldings, $N_{F}=30$, rate of channel utilization $U=0 .-0.3$, and beacon interval $=500 \mathrm{TU}$ ). 


\section{Evaluation}

We used an actual sensor node and WLAN APs to evaluate the basic performance of the AP-RSS measurement method in an office environment. Figure 10 depicts a prototype of our AP-RSS measurement system. The prototype consists of a Crossbow MICAz sensor node and a Panasonic CF-Y8 data-processing laptop. The MICAz sensor node is equipped with a Texas Instruments CC2420 ZigBee module. For WLAN APs, we prepared three Raspberry Pi devices equipped with a Buffalo WLI-UC-G301N WLAN module running on the OpenWrt operating system. We also prepared eight Netgear WNDR4300 WLAN APs also running on the OpenWrt operating system. To make the measurement easier, we implemented all the APRSS measurement processes presented in Sect. 3 on the data-processing laptop, not on the sensor node, except for RSS sampling.

\subsection{Preliminary experiment}

To determine the number of foldings in a channel-usage matrix, $N_{F}$, we conducted a preliminary experiment. We installed a sensor node and a WLAN AP separated by $2.5 \mathrm{~m}$ in an office environment, and collected the RSS samples on the sensor node every $128 \mu$ s for $1 \mathrm{~min}$. The WLAN AP's beacon interval was set to 109 TU. We folded the channel-usage samples calculated from the RSS samples at the beacon interval. We then extracted the rows of the channel-usage matrix by using a sliding window and evaluated the maximum overlapping rate for each windowed channel-usage matrix. We changed the window size, which corresponds to the number of foldings, $N_{F}$, from 5 to 100 .

Figure 11 depicts the distribution of the overlapping rates for $N_{F}$ ranging from 5 to 100 . The box for each $N_{F}$ represents the quartiles of the overlapping rates of all the trials, and the whiskers represent the rest of the distribution of the overlapping rates except for the outliers represented by data points. A notch on a box represents the median of the overlapping rates. Although $N_{F}$ should be minimized to reduce detection latency, a small $N_{F}$ results in outliers including large errors. We therefore determined the most suitable $N_{F}$ to be 30 , which, although, resulted in small variances of the overlapping rate, maintained the highest median overlapping rate.

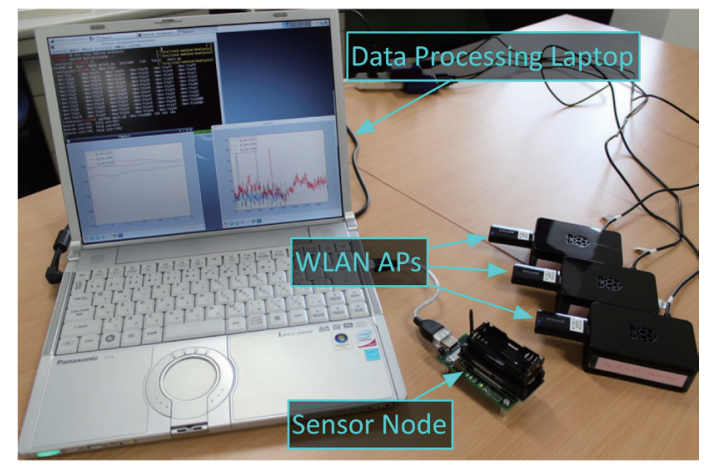

Fig. 10. (Color online) Prototype of AP-RSS measurement system. 


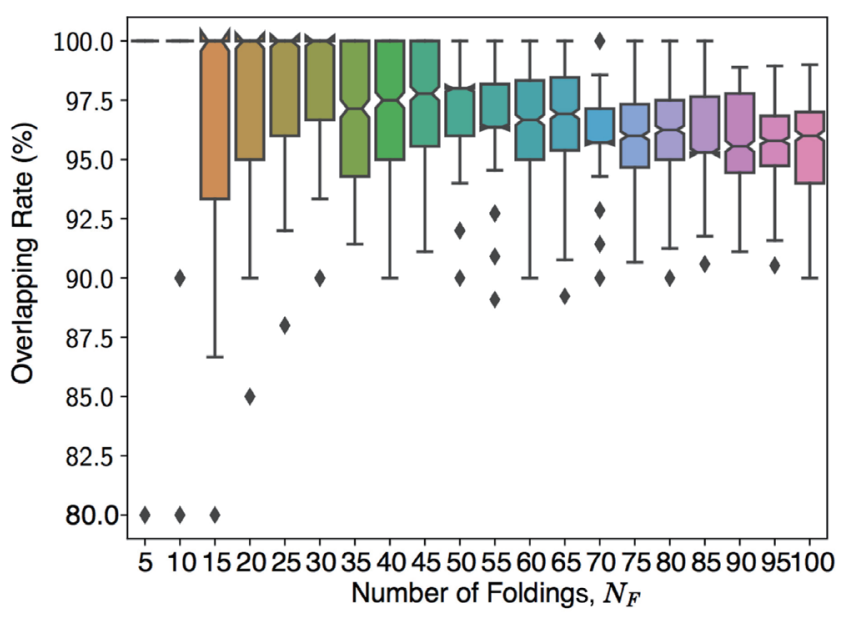

Fig. 11. (Color online) Distribution of overlapping rates for each number of foldings, $N_{F}$, in channel-usage matrix. Boxes and their notches represent quartiles and medians, respectively. Whiskers represent the rest of the distributions. Data points are outliers.

\subsection{Creation of beacon interval set}

Prior to performance evaluation, we created a set of beacon intervals by using the algorithm described in Sect. 3.2. The beacon intervals ranged between 50 and $500 \mathrm{TU}$, which is a practical range used in many WLAN APs available on the market. Furthermore, to create an initial set of beacon intervals, coprime numbers within this range were chosen. We collected RSS samples by using a sensor node and two APs separated by approximately $5 \mathrm{~m}$ in an office environment. We measured the FP detection rate $P_{f p}(A, B)$ while changing the combinations of beacon intervals of the APs. Finally, we created a set of 72 beacon intervals on the basis of the measured $P_{f p}(A, B)$.

Note that 30 beacon intervals are sufficient for practical deployment. We can create a set of 29 beacon intervals within the range of 50-200 TU. Moreover, we can use the same beacon interval on non-overlapping WLAN channels. By setting a sufficient distance between two WLAN APs to prevent the mixing of their signals, we can also reuse the same beacon interval in the same channel.

\subsection{Detection performance}

To demonstrate both AP detection and AP identification performance characteristics, we calculated true positive (TP) and FP detection rates. Let $I$ denote the created set of beacon intervals and $S(t)$ the beacon signals whose interval is $t \in I$. The TP belongs to the case in which $S(t)$ is detected as $S(t)$. The FP belongs to the case in which $S(u)$, for any $\{u \in I \mid u \neq t\}$, is detected as $S(t)$.

We installed a MICAz sensor node and eight WNDR4300 APs separated by approximately $5 \mathrm{~m}$ in an office environment. In each trial, eight different beacon intervals were randomly selected from the beacon interval set $I$ and were set to the APs. To calculate TP and FP 
detection rates, we collected the RSS samples by using the sensor node for 1 min and performed AP signal detection using the process in Sect. 3 for the eight beacon intervals. We repeated the trial 100 times and calculated the average of the TP and FP detection rates. Note that there were approximately 20 WLAN APs used for data communication in our experimental environment.

Figure 12 depicts the average TP and FP detection rates as a function of the beacon interval of the detection target AP. Figure 12 shows the following results:

(1) For all the target beacon intervals, the average TP detection rate was greater than $85 \%$. The minimum TP detection rate was $86.4 \%$ for the beacon interval of 449 TU. This result indicates that we can detect WLAN APs on sensor nodes more than $85 \%$ of the time.

(2) For all the target beacon intervals, the average FP detection rate was less than $20 \%$. The maximum FP detection rate was $19.8 \%$ for the beacon interval of 59 TU. The FP detection rate was worse than expected in Sect. 4. From Fig. 8, it can be seen that the maximum overlapping rate is approximately $10 \%$ when there are eight APs having beacon intervals greater than 50 TU. It can be observed from Fig. 9 that the overlapping rate threshold of $80 \%$ almost eliminates FP detection because of the WLAN data communication. Moreover, a large beacon time length might have increased the FP detection rate. We used beacon frames with a time length of $1472 \mu \mathrm{s}$, which is greater than $1 \mathrm{TU}(=1024 \mu \mathrm{s})$. The long beacon frames (in terms of their time length) appear in the same column in a channel-usage matrix more frequently than expected in the analysis, thereby increasing the FP detection rate.

(3) Target beacon intervals having a small time length tended to suffer from a high FP detection rate. The number of columns in a channel-usage matrix corresponds to the beacon interval of the detection target AP. When the number of columns in a channel-usage matrix is small, the number of columns occupied by a long beacon frame is significant, thereby resulting in the high FP detection rate.

The above-mentioned three results demonstrated that our AP-RSS measurement method successfully detected and identified sender APs with an FP detection rate less than 20\%. From the results of the analysis of the maximum overlapping rate caused by non-target APs in Sect. 4.1, we believe that we can derive similar results with up to 100 APs.

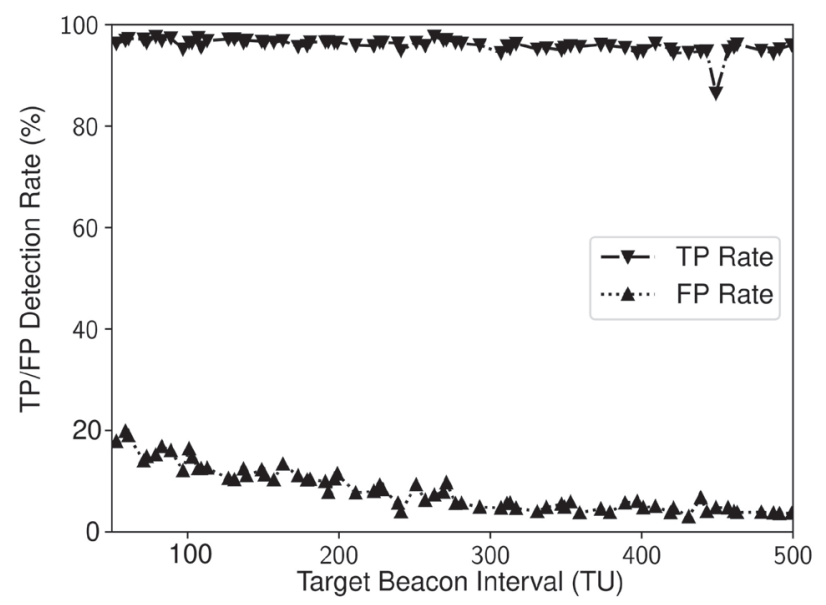

Fig. 12. Average TP and FP detection rates as a function of beacon interval of detection target AP. 


\subsection{RSS distribution}

To evaluate the AP-RSS error, we need to compare the AP-RSS with the true RSS, which is the RSS measured using a WLAN device installed immediately beside a sensor node. The RSS is unstable and changes frequently. The RSS measurement must be synchronized precisely within a few tens of microseconds for RSS comparison.

The experimental equipment we used, however, does not support precise synchronization. We therefore compared the RSS distributions in this paper. The two types of RSS must follow the same distribution but with different means and variances caused by the different wireless technologies used.

We installed a sensor node connected to the data-processing laptop equipped with a WLAN module and an AP separated by approximately $12 \mathrm{~m}$ in an office environment. The AP's beacon interval was 93 TU. In each trial, we sampled the AP-RSS for $4 \mathrm{~s}$ and calculated the average of the AP-RSS. The data-processing laptop also measured the RSS of the AP by using the pcap library (https://www.tcpdump.org) at the same time and calculated the average of the RSS. Note that the sampling timing was manually synchronized because of the limitation of the experimental equipment. We repeated the trial 1000 times and compared the distributions of the AP-RSS and true RSS.

Figure 13 depicts the distributions of both the AP-RSS and the true RSS. The blue dashed lines represent the medians. Figure 13 indicates the following:

(1) Both the AP-RSS and the true RSS seem to follow the Gaussian distribution except for outliers. The radio propagation environment changed frequently, resulting in the fluctuations of the RSS. The standard deviations of the AP-RSS and true RSS were 1.36 and $0.98 \mathrm{~dB}$, respectively. The ZigBee sensor uses a $2 \mathrm{MHz}$ band, while the WLAN sensor uses a $22 \mathrm{MHz}$ band. The AP-RSS derived using the narrow-band ZigBee sensor node was more affected by the environmental changes than the RSS derived using the WLAN sensor.

(2) The medians of the AP-RSS and true RSS were -48.0 and $-44.9 \mathrm{dBm}$, respectively. By comparing the medians, an offset can be confirmed between the RSS measurements

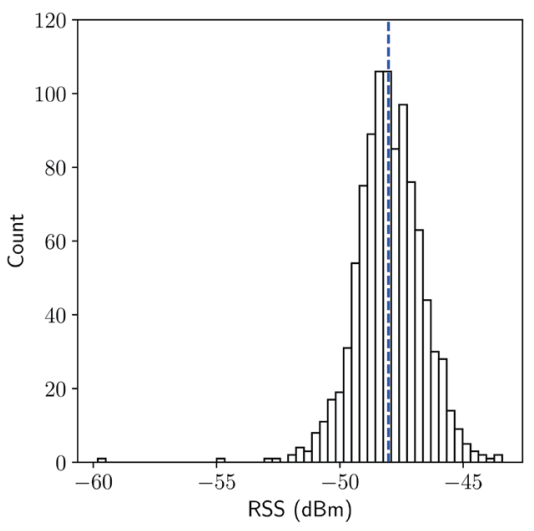

(a)

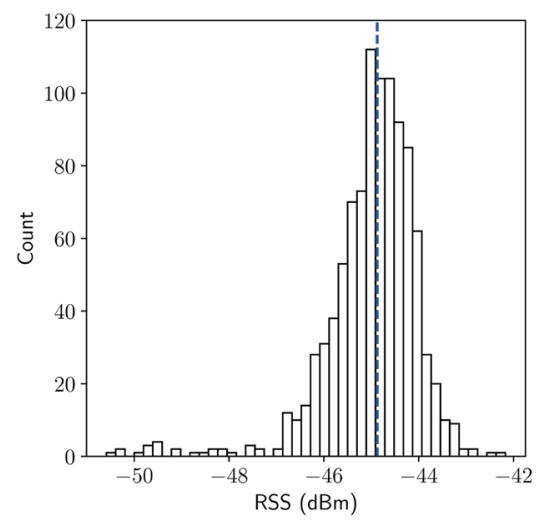

(b)

Fig. 13 (Color online) Distributions of both AP-RSS and true RSS. The blue dashed lines represent the medians. 
obtained using the ZigBee and WLAN sensors. The offset was mainly caused by the differences in bandwidth and antenna gain between the sensors.

As described above, the AP-RSS and true RSS had different medians and standard deviations caused by the different wireless technologies used. We normalized the RSS distributions before comparing them: each RSS set was transformed into a new set with a median of 0 and a standard deviation of 1 .

We performed the Kolmogorov-Smirnov test on the normalized RSS sets. The $p$-value was calculated to be 0.33 . We cannot confirm that the distributions of the AP-RSS and true RSS were different at a significance level of $p<0.05$ upon ignoring the offset and difference in variation.

\section{Related Work}

Studies on indoor localization have mainly investigated deployment-cost reduction and accuracy improvement. Although a WLAN system was targeted in most of the studies, the methods presented therein supported ZigBee sensors.

Iterative multilateration ${ }^{(6)}$ is one of the popular localization technologies that reduce the number of initial anchor nodes. In iterative multilateration, nodes whose location is estimated are used as new anchors. The number of initial anchor nodes can also be reduced by anchor location optimization. ${ }^{(7)}$ However, to reduce the localization errors in a large localization target area, we still need to deploy many initial anchor nodes.

Fingerprinting localization with a crowdsourced site survey, ${ }^{(8-15)}$ which collects the RSS everywhere in a localization target area for fingerprinting, eliminates the need for anchor installation. For sensor localization, there are a few cooperators that always carry a sensor node.

For accuracy improvement, many methods ${ }^{(16-21)}$ such as multilateration by employing geometrical features $^{(16)}$ and fingerprinting by employing physical layer information ${ }^{(20)}$ have been reported. These methods are useful for improving the accuracy of our sensor localization system.

ZiFind $^{(27)}$ is a fingerprinting localization method that uses WLAN APs for ZigBee sensor localization. ZiFind utilizes beacon transmission timing information as a feature in fingerprinting localization. Although ZiFind requires no anchor ZigBee sensor node, special WLAN devices, called ZiFind mappers, need to be installed at known locations. Moreover, ZIL $^{(28)}$ is another fingerprinting localization method that uses the RSS and transmission timing of WLAN beacon signals. In the ZIL method, site survey, i.e., data collection using a sensor node, is mandatory, which has a large cost in large-scale sensor networks.

\section{Conclusion}

In this paper, we presented a sensor localization system that eliminates the deployment of new anchors. The key idea is to utilize the WLAN APs already installed in the environment as anchors. However, the sensor nodes in the system are equipped with a ZigBee module, 
which cannot detect WLAN signals. We therefore developed a cross-technology signal detection method and designed an AP-RSS measurement method, both of which will be the key technology in our sensor localization system. We also presented a simple AP identification method based on AP beacon intervals to identify the sender APs. The experimental evaluations revealed that the AP-RSS measurement method identified the sender APs with an FP detection rate less than 20\%. Moreover, the measured AP-RSS followed the same distribution as the RSS measured using a WLAN device with an offset and a different standard deviation. As future work, we need to confirm that we can perform sensor localization using the measured AP-RSS.

\section{Acknowledgments}

This work was supported in part by the Japan Society for the Promotion of Science (JSPS) KAKENHI Grant Numbers JP15H05708, JP17H01741, and JP18K18041.

\section{References}

1 S. Ishida, K. Izumi, S. Tagashira, and A. Fukuda: Proc. 82nd Vehicular Technology Conf. (VTC-Fall) (IEEE, 2015) 1. https://doi.org/10.1109/VTCFall.2015.7391191

2 K. Izumi, S. Ishida, S. Tagashira, and A. Fukuda: Proc. 3rd Int. Symp. Computing and Networking (CANDAR) (IPSJ, 2015) 115. https://doi.org/10.1109/CANDAR.2015.54

3 J. Wang, R. K. Ghosh, and S. K. Das: J. Control Theory Appl. 8 (2010) 2. https://doi.org/10.1007/s11768-0109187-7

4 L. Cheng, C. Wu, Y. Zhang, H. Wu, M. Li, and C. Maple: Int. J. Distrib. Sens. Netw. 8 (2012) 962523. https:// doi.org/10.1155/2012/962523

5 Á. Lédeczi and M. Maróti: Philos. Trans. R. Soc. London, Ser. A 370 (2012) 85. https://doi.org/10.1098/ rsta.2011.0216

6 M. Minami, Y. Fukuju, K. Hirasawa, S. Yokohama, M. Mizumachi, H. Morikawa, and T. Aoyama: UbiComp 2004: Ubiquitous Computing. UbiComp 2004. Lecture Notes in Computer Science, Vol. 3205, N. Davies, E. D. Mynatt, and I. Siio, Eds. (Springer, Heidelberg, 2004) pp. 347-365. https://doi.org/10.1007/978-3-540-301196_21

7 L. Huang, F. Wang, C. Ma, and W. Duan: Advances in Wireless Sensor Networks. CWSN 2012. Communications in Computer and Information Science, Vol. 334, R. Wang and F. Xiao, Eds. (Springer, Heidelberg, 2013) pp. 117-126. https://doi.org/10.1007/978-3-642-36252-1_12

8 P. Bolliger: Proc. Int. Workshop on Mobile Entity Localization and Tracking in GPS-less Environments (MELT) (ACM, 2008) 55. https://doi.rg/10.1145/1410012.1410025

9 A. Barry, B. Fisher, and M. L. Chang: Mobile Entity Localization and Tracking in GPS-less Environnments. MELT 2009. Lecture Notes in Computer Science, Vol. 5801, R. Fuller and X. D. Koutsoukos, Eds. (Springer, Heidelberg, 2009) pp. 197-212. https://doi.org/10.1007/978-3-642-04385-7_14

10 J.-G. Park, B. Charrow, D. Curtis, J. Battat, E. Minkov, J. Hicks, S. Teller, and J. Ledlie: Proc. 8th Int. Conf. Mobile Systems, Applications, and Services (MobiSys) (ACM, 2010) 271. https://doi. org/10.1145/1814433.1814461

11 A. Rai, K. K. Chintalapudi, V. N. Padmanabhan, and R. Sen: Proc. 18th Annu. Int. Conf. Mobile Computing and Networking (MobiCom) (ACM, 2012) 293. https://doi.org/10.1145/2348543.2348580

12 H. Wang, S. Sen, A. Elgohary, M. Farid, M. Youssef, and R. R. Choudhury: Proc. 10th Int. Conf. Mobile Systems, Applications, and Services (MobiSys) (ACM, 2012) 197. https://doi.org/10.1145/2307636.2307655

13 Z. Yang, C. Wu, and Y. Liu: Proc. 18th Annu. Int. Conf. Mobile Computing and Networking (MobiCom) (ACM, 2012) 269. https://doi.org/10.1145/2348543.2348578

14 C. Wu, Z. Yang, Y. Liu, and W. Xi: IEEE Trans. Parallel Distrib. Syst. 24 (IEEE, 2013) 839. https://doi. org/10.1109/TPDS.2012.179

15 Z. Jiang, J. Zhao, J. Han, Z. Wang, S. Tang, J. Zhao, and W. Xi: Proc. 10th Int. Conf. Mobile Ad-Hoc and Sensor Systems (MASS) (IEEE, 2013) 384. https://doi.org/10.1109/MASS.2013.84

16 G. S. Kuruoglu, M. Erol, and S. Oktug: Proc. Global Telecommun. Conf. (GLOBECOM) Workshops (IEEE, 2009) 1. https://doi.org/10.1109/GLOCOMW.2009.5360717 
17 A. W. Tsui, Y.-H. Chuang, and H.-H. Chu: Mob. Netw. Appl. 14 (2009) 677. https://doi.org/10.1007/s11036008-0139-0

18 A. Kushki, K. N. Plataniotis, and A. N. Venetsanopoulos: Trans. Mob. Comput. 9 (2010) 405. https://doi. org/10.1109/TMC.2009.141

19 K. Kaemarungsi and P. Krishnamurthy: Pervasive Mob. Comput. 8 (2012) 292. https://doi.org/10.1016/ j.pmcj.2011.09.003

20 S. Sen, B. Radunovic, R. R. Choudhury, and T. Minka: Proc. 10th Int. Conf. Mobile Systems, Applications, and Services (MobiSys) (ACM, 2012) 183. https://doi.org/10.1145/2307636.2307654

21 N. Wirström, P. Misra, and T. Voigt: Proc. Annu. Conf. Wireless On-demand Network Systems and Services (WONS) (IEEE, 2014) 25. https://doi.org/10.1109/WONS.2014.6814718

22 R. Zhou, Y. Xiong, G. Xing, L. Sun, and J. Ma: Proc. 16th Annu. Int. Conf. Mobile Computing and Networking (MobiCom) (ACM, 2010) 49. https://doi.org/10.1145/1859995.1860002

23 Texas Instruments: CC2420 datasheet: https://www.ti.com/ (accessed January 2015)

24 S. M. Kim and T. He: Proc. 21st Annu. Int. Conf. Mobile Computing and Networking (MobiCom) (ACM, 2015) 317. https://doi.org/10.1145/2789168.2790098

25 S. M. Kim, S. Ishida, S. Wang, and T. He: Trans. Networking 25 (2017) 2974. https://doi.org/10.1109/ TNET.2017.2724539

26 IEEE Standards Association: IEEE Standard. https://standards.ieee.org/

27 Y. Gao, J. Niu, R. Zhou, and G. Xing: Proc. INFOCOM (IEEE, 2013) 2940. https://doi.org/10.1109/ INFCOM.2013.6567105

28 J. Niu, B. Wang, L. Shu, T. Q. Duong, and Y. Chen: IEEE J. Sel. Areas Commun. 33 (2015) 1431. https:/doi. org/10.1109/JSAC.2015.2430171

\section{About the Authors}

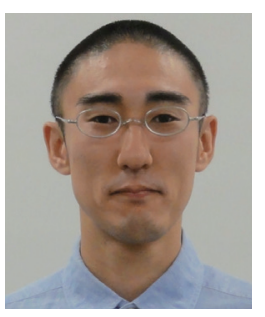

Shigemi Ishida received his B.E. degree in electronic engineering from Shibaura Institute of Technology, Tokyo, Japan, in 2006 and his M.Sc. degree in information science and Ph.D. degree in electrical engineering, from the University of Tokyo, Tokyo, Japan, in 2008 and 2012, respectively. From 2008 to 2009, he was a programmer in industry. From 2011 to 2013, he was a Japan Society for the Promotion of Science Research Fellow. In 2013, he became a visiting scholar with the Department of Computer Science and Engineering, University of Minnesota, Minneapolis, MN, USA. $\mathrm{He}$ is currently an assistant professor with the Faculty of Information Science and Electrical Engineering, Kyushu University, Fukuoka, Japan. His research interests include wireless sensor networks, low-power wireless communications, localization systems, cross-technology communications, and intelligent transport systems. He is currently focused on localization systems and sensing technologies for the better understanding of the world around us. He is a member of the IEICE, IPSJ, and IEEE.

(ishida@ait.kyushu-u.ac.jp)

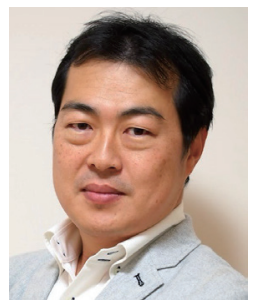

Yutaka Arakawa was born in 1977. He received his B.E., M.E., and Ph.D. degrees from Keio University, Tokyo, Japan, in 2001, 2003, and 2006, respectively. He was an assistant professor with Keio University from 2006 to 2009 and with Kyushu University, Fukuoka, Japan, from 2009 to 2013. After working as an associate professor with Nara Institute of Science and Technology from 2013 to 2018, he became a professor with the Graduate School of Information Science and Electrical Engineering, 
Kyushu University, in 2019. Additionally, he was a visiting researcher with ENSEEIHT (France) in 2011, DFKI (Germany) in 2012, and UCLA (USA) in 2018. He was the recipient of the IPSJ Yamashita SIG Research Award in 2011, the 24th Hiroshi Ando Memorial Award in 2011, the second prize in the mobile app competition held in MobiCom2014, the IPSJ Nagao Special Researcher Award in 2015, the UbiComp/ISWC Best Demo Award in 2016, the IPSJ/IEEE-CS Young Computer Researcher Award in 2018, and the IEEE PerCom Best Demonstration Award in 2019. His current research interests include participatory sensing, location-based information systems, activity recognition, and behavior change support systems. He is a member of the IEICE, IPSJ, IEEE, and ACM. (arakawa@ait.kyushu-u.ac.jp)

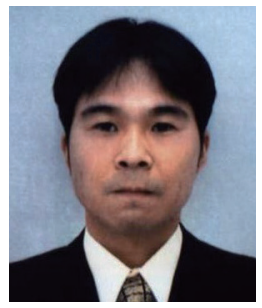

Shigeaki Tagashira received his B.Eng. degree from Ryukoku University, Kyoto, Japan, in 1996 and his M.Eng. and D.Eng. degrees in information science from Nara Institute of Science and Technology, Ikoma, Japan, in 1998 and 2000, respectively. He was a research associate with Hiroshima University, Hiroshima, Japan, from 2000 to 2007. In 2007, he joined Kyushu University, Fukuoka, Japan, as a project associate professor. Since 2014, he has been a professor with Kansai University, Suita, Japan. His current research interests include ubiquitous/mobile computing and system software. $\mathrm{He}$ is a member of the IEICE, IPSJ, and IEEE.

(shige@res.kutc.kansai-u.ac.jp)

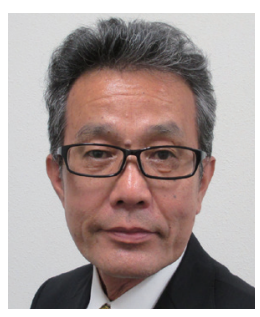

Akira Fukuda received his B.Eng., M.Eng., and Ph.D. degrees in computer science and communication engineering from Kyushu University, Fukuoka, Japan, in 1977, 1979, and 1985, respectively. From 1977 to 1981, he was with Nippon Telegraph and Telephone Corporation, where he evaluated the performance of computer systems and queueing theory. From 1981 to 1991 and from 1991 to 1993, he was with the Department of Information Systems and the Department of Computer Science and Communication Engineering, Kyushu University, respectively. In 1994, he joined Nara Institute of Science and Technology, Ikoma, Japan, as a professor. He has been a professor with the Graduate School of Information Science and Electrical Engineering since 2001, a director with the System LSI Research Center since 2008, a distinguished professor since 2015, and a director with the R\&D Center for Smart Mobility since 2016, all with Kyushu University. His research interests include embedded systems, ubiquitous computing, system software (operating systems, compilers, and run-time systems), parallel and distributed systems, and performance evaluation. He is an IPSJ fellow. He is a member of the ACM, the IEEE Computer Society, the IEICE, the IPSJ, and the Operations Research Society of Japan. (fukuda@ait.kyushu-u.ac.jp) 